\title{
Structural design, development and qualification tests of grid fin
}

\author{
Able C. Kurian*, B. Santhosh, P. D. Shaji, Sibi Joseph and A. Rajarajan \\ Composite Launch Vehicle Structures Group, Composites Entity, Vikram Sarabhai Space Centre, Thiruvananthapuram 695 013, India
}

Crew Escape System (CES) is one of the most critical subsystems in a human-rated launch vehicle. It is an emergency escape module to rescue the Crew Module (CM) with the crew to a safe distance from the launch vehicle in an exigency at the launch pad or during atmospheric phase. Pad Abort Test (PAT) is a technology demonstration on the capability of CES to evacuate the crew in case of any exigency at the launch pad, the first in a series of tests to qualify a CES, which is a crucial technology relevant for human spaceflight. There is a wide range of altitude-Mach number regime over which CES is expected to operate and deliver the required performance. Most important among them is the requirement of aerodynamic stability for the entire Mach number regime. The configuration of CES called for an additional lift producing surfaces at its bottom to get the required stability conditions. Accordingly, four grid fins are located at the aft end of CES to move the centre of pressure down for better stability. Grid fin is a lattice structure of several aerodynamic surfaces supported by rectangular outer frames. It is designed for a variable pressure load acting on the fin elements and outer frames. Grid fins are designed and developed with composite material because of its high specific stiffness and specific strength. Composite grid fins are designed, developed, qualified and successfully flown in PAT flight. This article presents the design methodology, development, validation through structural acceptance and deployment tests of composite grid fins meant for the PAT of CES.

Keywords: Composite materials, Crew Escape System, Crew Module, grid fin, Pad Abort Test.

\section{Introduction}

COMPOSITE materials are widely used in launch vehicle structures due to their high specific strength and specific stiffness. Composite grid fins are used for providing aerodynamic stability to the Crew Escape System (CES) during its trajectory after separation from the launch vehicle in case of an exigency. Grid fins have much shorter 'chord' than conventional planar fins and they generate low hinge moments, thereby reducing the size of

*For correspondence. (e-mail: able_c_kurian@vssc.gov.in) actuators. Grid fin configuration was optimized and drag was tailored with minimum impact on the aerodynamic properties through suitable lattice control design ${ }^{1}$. Four grid fins are mounted at the aft end of CES. Grid fins are initially in stowed condition until CES is activated. They are deployed using pyro device with a total deployment time of $525 \mathrm{~ms}$, when CES is activated in case of exigency. A thermal protection layer of epoxy paint with about $0.9 \mathrm{~mm}$ thickness is provided on the grid fins to maintain the base structure within allowable temperature limit of $120^{\circ} \mathrm{C}$.

Grid fin is a cantilever structure in deployed condition with an overall size of about $1.8 \mathrm{~m} \times 1.5 \mathrm{~m}$. It forms a lattice structure of several aerodynamic surfaces called fin elements supported by rectangular outer frames. Fin elements are composite monocoque hollow sections of uniform depth interconnected by metallic inserts. Bonded joints are provided between the carbon fiber reinforced plastic (CFRP) fin elements and metallic inserts. Outer frames are composite sandwich material of varying depth. Fin element sub-assembly is assembled to outer frames using bonded and bolted joints with metallic brackets. A three-point holding system is provided for each grid fin in stowed condition. At the bottom, grid fins are hinged to the Crew Module Fairing (CMF) through two hinge brackets at extreme locations, and on the top the fin is connected to CMF through a hold and release-cumthrusting device. Hydraulic dampers are provided on either side of the grid fin outer frame, to reduce impact energy at the time of full deployment within the specified time and to lock the fin after deployment.

Composite sandwich gussets are provided for better stiffness at the hinge interfaces of the grid fin. Composite

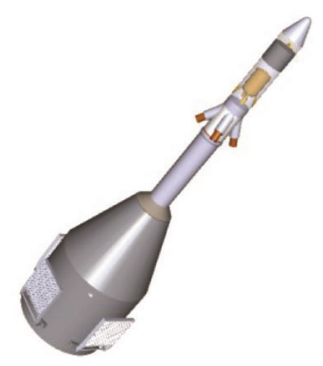

Grid fin Stowed condition

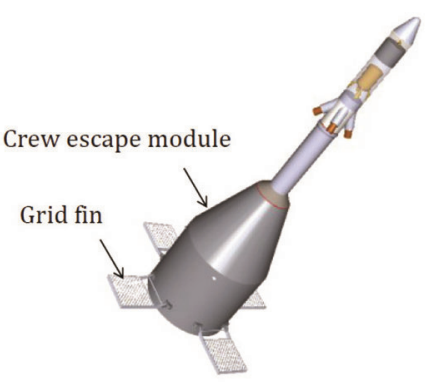

Grid fin Deployed condition
Figure 1. Overall configuration of Human Spaceflight Programme (HSP) Crew Escape System (CES). 
sandwich outer frame at the hinge and damper interfaces are embedded with thick CFRP laminates to meet edge shear requirement and enhance stress capability. Grid fin outer frame at the actuator bracket interface is made stiffened similar to box-type construction by embedding CFRP channels along its length and providing additional reinforcement layers on the outer face skin. Figures 1 and 2 show the overall configuration of the Human Spaceflight Programme (HSP) CES and geometrical configuration of the grid fin respectively. Figures 3 and 4 show the interfaces of grid fin in stowed and deployed condition respectively.

\section{Design-driven events}

Grid fins are initially kept in stowed condition and in the event of launch abort when CES activation is demanded,
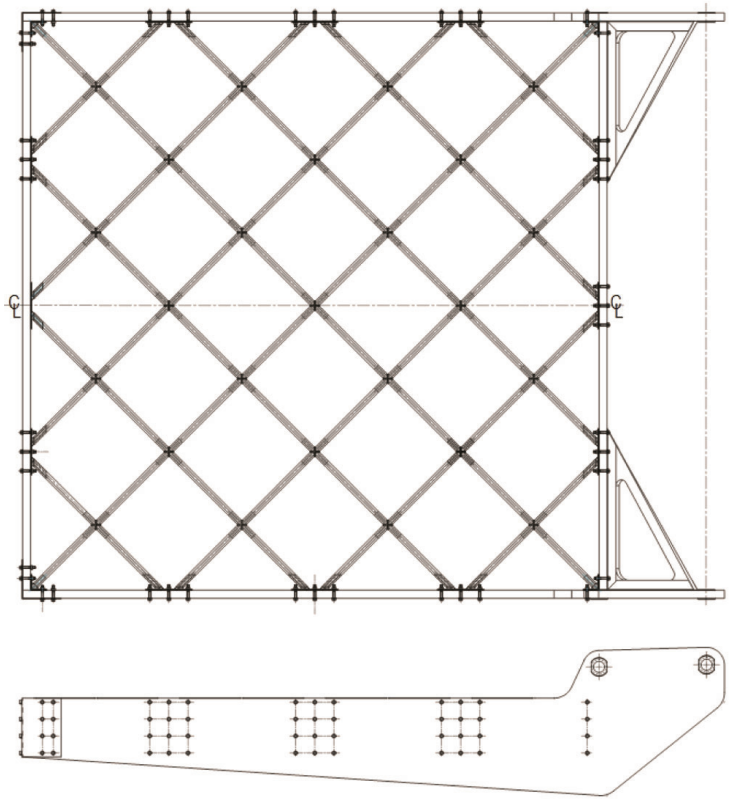

Figure 2. Grid fin configuration.
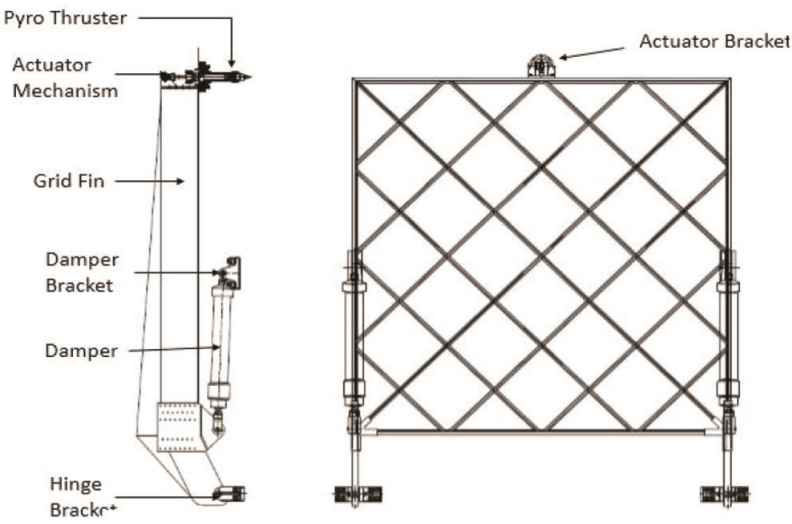

Figure 3. Interfaces of grid fin in stowed condition. all the four grid fins are to be deployed simultaneously within a stipulated time frame on a single command. The deployment system consists of a pyro-based actuation system designed to provide initial locking and preloading to the grid fin in stowed condition and to impart an angular velocity during actuation; and two hydraulic dampers designed to absorb the kinetic energy imparted by pyro thruster, to reduce impact energy during the final phase of deployment and to lock the fins in deployed condition during the entire flight regime using a collet-based locking system. The peak force experienced during pyro actuation is $70 \mathrm{kN}$ and the duration of the force is $13 \mathrm{~ms}$. Figure 5 shows pyro actuation load versus time plot. The transient energy released during pyro actuation induces very large local loads on fin hold down interface making

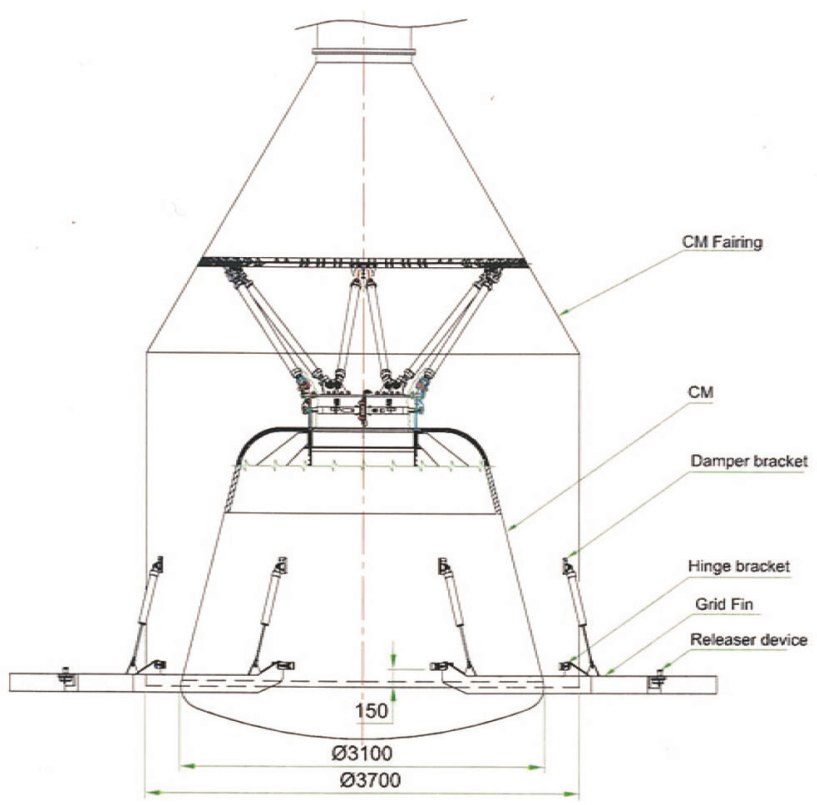

Figure 4. Interfaces of grid fin in deployed condition.

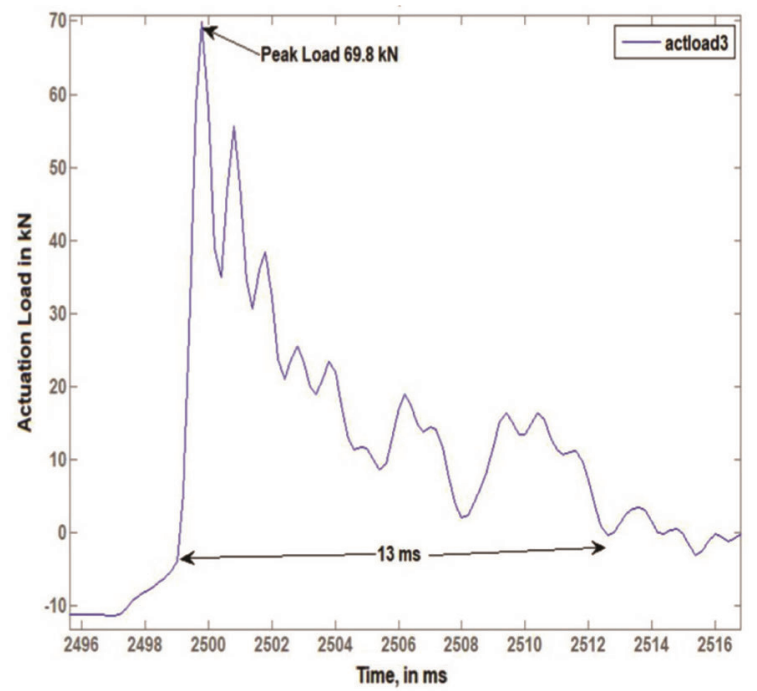

Figure 5. Pyro actuation load versus time plot.

CURRENT SCIENCE, VOL. 120, NO. 1, 10 JANUARY 2021 
its structural design more complex. During launch abort while the grid fins are in the deployed condition, the fin structure will be subjected to CES flight loads which include axial loads, shear load, bending moment and aero dynamic pressure imparting huge reaction loads at damper and hinge interfaces.

Composite grid fin is designed with sufficient strength and stiffness to function satisfactorily under the impact loads during the initial and final phases of deployment within specified time without affecting the stability of CES. Also, it should perform satisfactorily under flight loads in deployed condition without any failure. The maximum deflection at the tip of the fin is limited so that adverse effects of the loads on the fin during flight are eliminated.

\section{Methodology of load estimation}

CES is expected to operate under a widely varying range of altitudes and Mach numbers in a manned mission, including activation from the launch pad. Critical load cases for the grid fin are identified among the entire Mach number regime. Aerodynamic pressure distribution on grid fin in deployed condition for these critical load cases together with acceleration levels are considered in the design.

The structural ultimate loads acting on the grid fin during flight are used for the design. Since all the four fins are subjected to loads of different magnitude and direction, an extensive analysis is required for design verification. Grid fin design for flight load is basically governed by maximum normal load acting on its surface. The maximum value of ultimate equivalent normal load among different grid fins is $46 \mathrm{kN}$.

\section{Challenges in grid fin design}

Grid fin is an assemblage of 64 fin elements (aerofoil sections) within a square framework. The primary challenge was to design the structure which ensures feasibility of processing/assembly. Grid fin outer frames were designed as CFRP sandwich structure with high-strength carbon/ epoxy face skin and aluminium alloy honeycomb core. Monocoque, thin-walled, slender fin elements were designed with high-strength carbon/epoxy material after ensuring the proof of concept using a split mould. The lay-up was selected to meet the stiffness and strength requirements based on classical lamination theory ${ }^{2,3}$. Layup sequence of $0^{\circ} / \pm 45^{\circ}$ combination was selected maintaining balance and symmetry to avoid bending/warping during curing/loading. Detailed finite element (FE) analysis has been carried out using general purpose FE software Nastran for static, buckling and frequency requirements. Figure 6 shows the FE model of grid fin and Figure 7 shows the displacement plot. Maximum fibre direction tensile stress observed in the static analysis of grid fin in deployed condition was $542 \mathrm{MPa}$, whereas the compressive stress observed was $-483 \mathrm{MPa}$. Margin of safety in static analysis is 2 against the compressive strength of CFRP and 2.8 against tensile strength of CFRP. Buckling analysis showed a minimum buckling load factor of 1.4 against non uniform aerodynamic pressure. This design gives a first-mode frequency of $30 \mathrm{~Hz}$ in out-of-plane bending and second mode frequency of $56 \mathrm{~Hz}$ in in-plane bending with appropriate boundary conditions at hinge and damper interfaces.

Pyro thruster loads act locally on grid fin actuator bracket interface at the initial phase of deployment. The specified action time for the pyro thruster load is $13 \mathrm{~ms}$. The peak load experienced at the hold-down interface during pyro deployment is $70 \mathrm{kN}$. Grid fin structure has to withstand these shock loads without affecting the

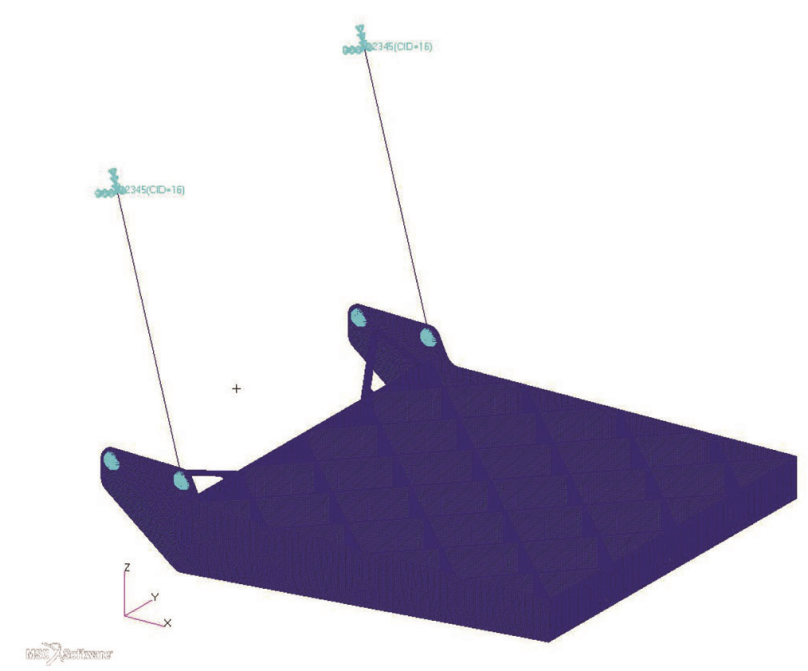

Figure 6. Grid fin finite element model.

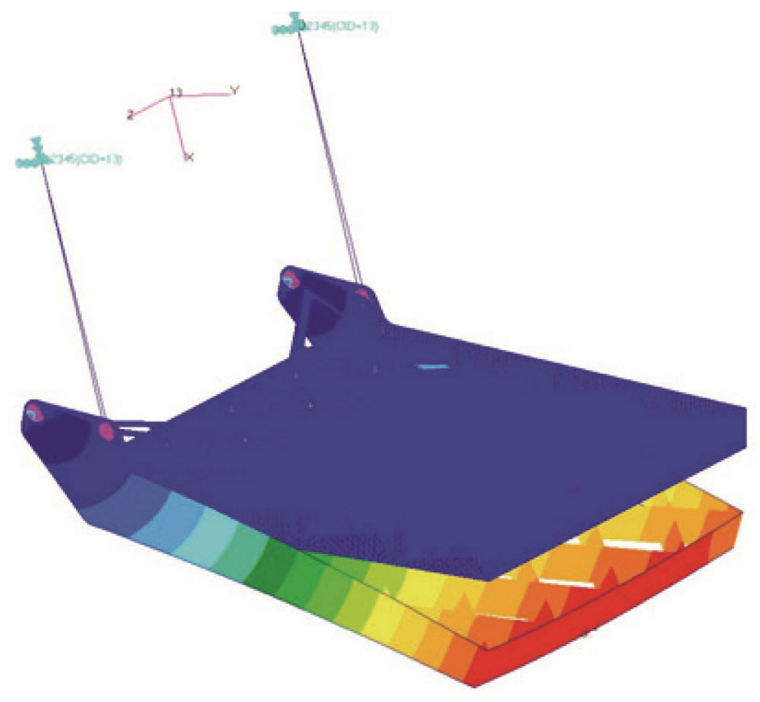

Figure 7. Displacement plot of grid fin. 
stability of CES. Design of actuator bracket interface on grid fin was the most challenging task during the design and development phase. Grid fin outer frame is made stiffened similar to box-type construction by embedding CFRP channels along its length and additional reinforcement layers are provided on the outer face skin. Outer frame local deformation at actuator bracket interface is eliminated by rigidizing the honeycomb core locally. In addition, fasteners are provided at the bonded interfaces of metallic brackets and fin elements adjacent to the actuator bracket, to efficiently transfer the shear loads generated by the pyro deployment loads.

Total deployment time of grid fin is estimated as $525 \mathrm{~ms}$. The impact energy occurring during the final phase of deployment induces very large local loads on the hinge and damper interfaces of the grid fin. Design of these interfaces is further controlled by the flight loads. Maximum interface loads in grid fin deployed condition for the ultimate flight load was $101 \mathrm{kN}$, which was estimated at damper locations. Limited edge distance on grid fin which is governed by the design requirements of hinge and damper brackets makes the design of these interfaces more critical. Thick CFRP laminates were processed through press moulding and face machining was carried out to have better dimensional tolerance levels. These machined laminates were embedded inside the composite sandwich outer frames at hinge and damper interfaces to meet edge shear requirement ${ }^{4}$ and to enhance stress capability. Specimen-level tests were carried out to validate the interface design and establish the margins. A minimum margin of 0.9 is ensured in the design of damper and hinge interfaces.

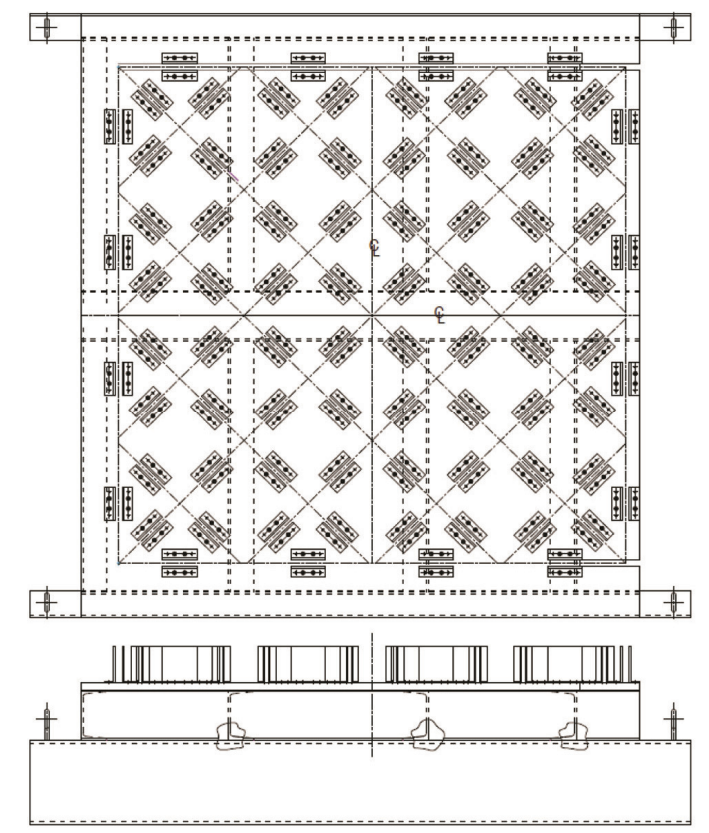

Figure 8. Assembly fixture for grid fin.
Titanium bushes are provided at the hinge and damper interfaces for improved bearing capability and for better dimensional tolerance levels. Grid fin is attached to the hinge bracket through hinge bolt with hinge bush. The bolt will preload the hinge bush within the bracket and the grid fin will be free to rotate at this condition. Interface machining was carried out on grid fin assembly level to ensure the colinearity of hinge and damper interfaces. Interface machining helped to keep the overall interface dimensions of grid fin within acceptable limits for ensuring its smooth assembly and deployment.

The prepreg layers have been cut in desired orientation and configuration in prepreg cutting machine with minimum wastage of material. Metallic split moulds are employed for the processing of thin-walled, slender, monocoque fin elements for easiness of extraction. The prepreg layers and honeycomb core for sandwich panels were laid over the metallic surface plate at desired orientations and co-cured at $175^{\circ} \mathrm{C}$ in autoclave under vacuum.

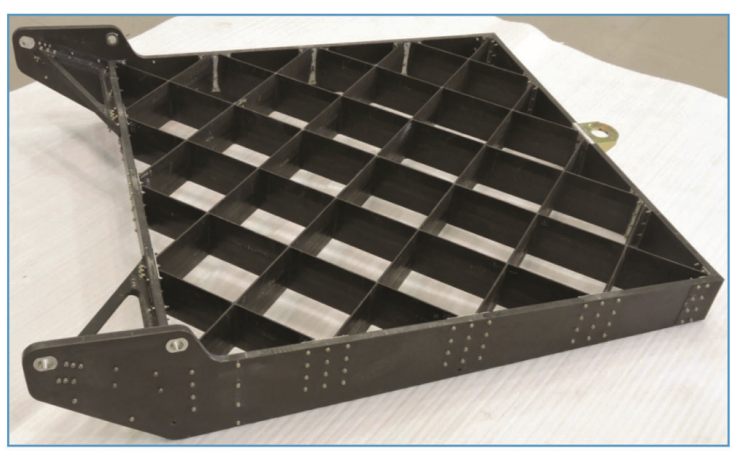

Figure 9. Composite grid fin - realized hardware.

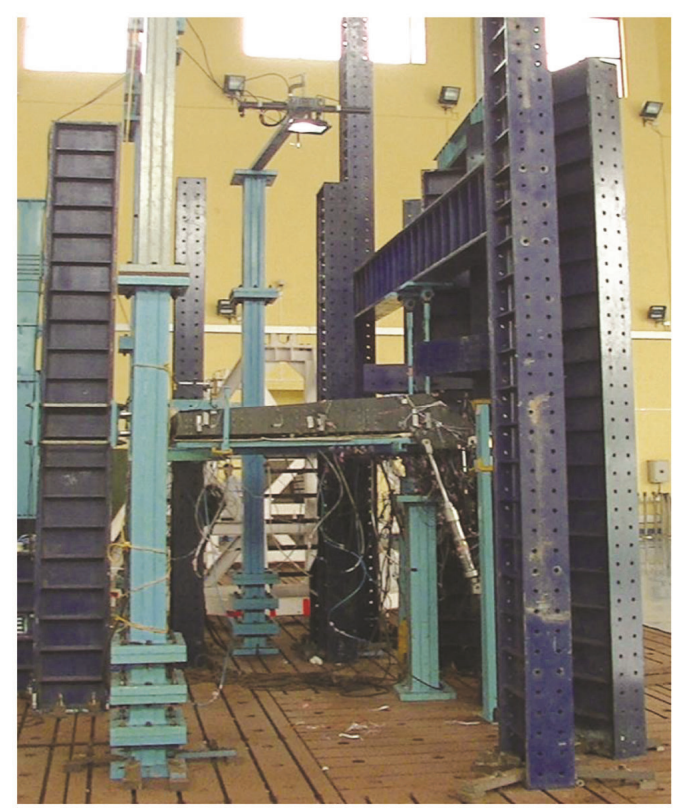

Figure 10. Structural test set-up. 

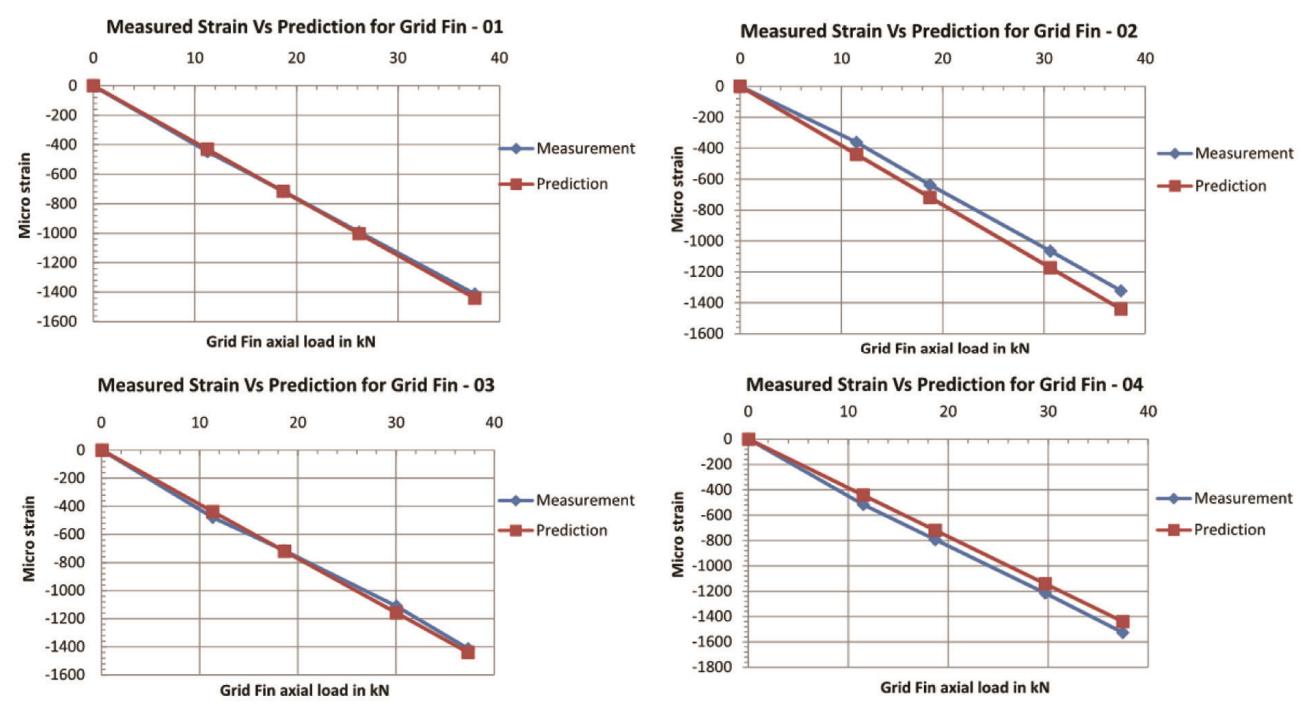

Figure 11. Comparison of strain during structural test.
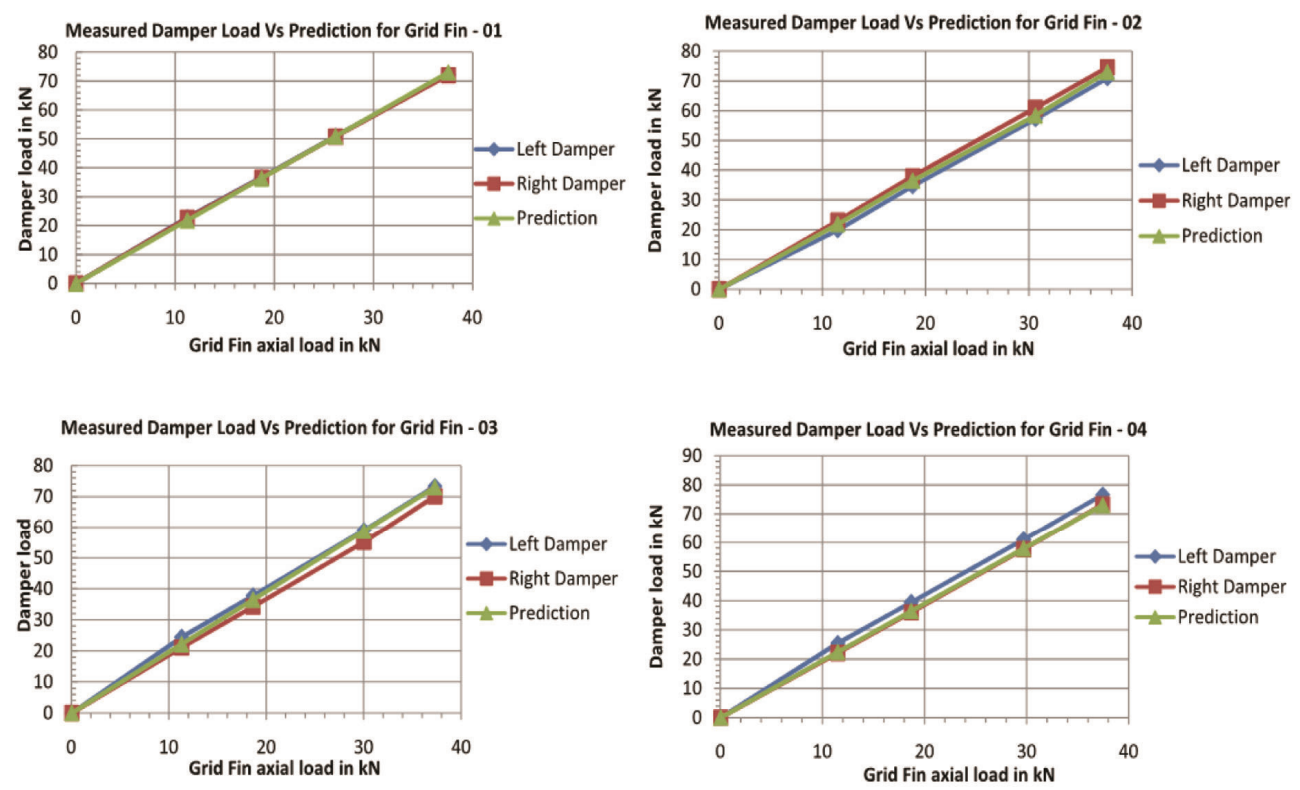

Figure 12. Comparison of damper loads during structural test.

All the grid fin outer frames were cut from bigger size composite sandwich panels realized. CFRP fin elements and outer frames are assembled using a dedicated fixture to meet the dimensional requirements. Figure 8 shows the assembly fixture for grid fin. This design meets the strength and stiffness requirements for all the design critical events. Figure 9 shows the composite grid fin realized. The mass of the realized grid fin is $60 \mathrm{~kg}$.

\section{Qualification tests}

The primary objectives of the structural acceptance test are to assess the real performance of the structure and to validate the design. Structural acceptance testing of grid fin necessitates application of normal load and in-plane load at different locations according to the design. The structural acceptance testing of grid fin was carried out at unit level on ground supports. The normal loads and in-plane loads are applied using pressure chambers. Figure 10 shows the structural test set-up of the grid fin.

Displacements and strains on the grid fin were predicted for validation of the test article. Composite grid fin is tested for the design-proof load. Measured displacements and strain values on the grid fin were compared with the predicted values. Strains and displacements on the grid fin showed a linear trend and matched well with the predictions. Maximum tensile strain measured along 
the length of the grid fin outer frame among four hardware ranges from 1501 to $1966 \mu$ s, against the prediction of $1895 \mu \mathrm{s}$. Maximum compressive strain measured along the length of outer frame ranges from -1703 to $-1962 \mu \mathrm{s}$, against the prediction of $-1748 \mu \mathrm{s}$. Figure 11 shows strain comparison for a typical strain gauge. Deflection of grid fin was mainly controlled by the stiffness of hydraulic dampers. Maximum axial deflection observed at the tip of the grid fin ranges from 46.8 to $54.6 \mathrm{~mm}$, against the prediction of $46.7 \mathrm{~mm}$. The increase in tip deflection was because of the rigid body displacement contributed by the deployment system due to the clearances provided in the hydraulic damper locking mechanism. Based on this test observation, a spacer was provided in the damper assembly to reduce the clearance. Loads experienced by the dampers were also measured during test and compared with predicted values. Maximum load measured on grid fin damper during test was $97.7 \mathrm{kN}$. All the four grid fins withstood the acceptance loads successfully. Composite grid fin successfully underwent integrated-level axial load test along with CMF to assess the integrated level performance of the system and to qualify the interfaces. Figure 12 shows a comparison of measured damper load and prediction during grid fin unit-level structural test.

The grid fin underwent hydraulic deployment test and pyro deployment test as a part of its functional

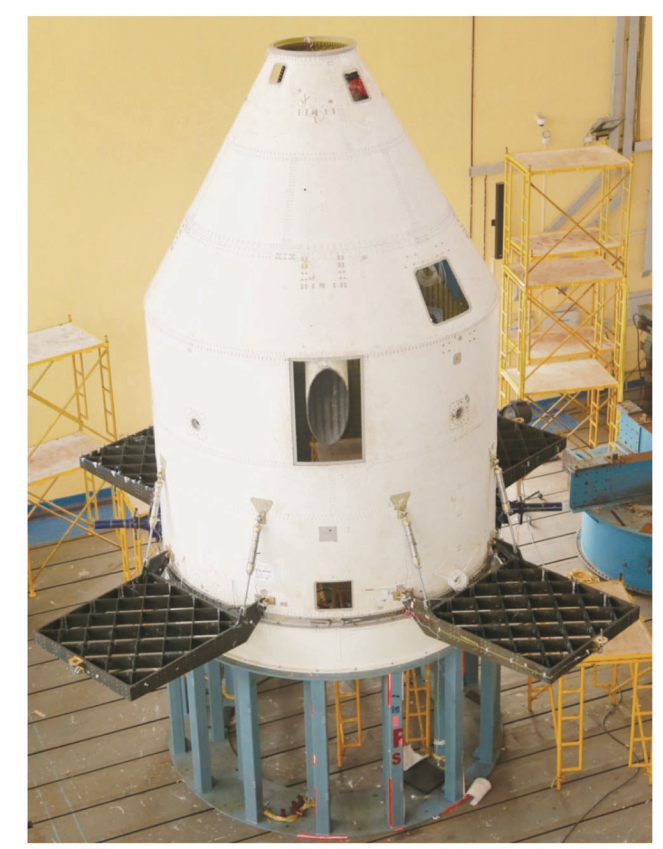

Figure 13. Crew Module Fairing with composite grid fins in deployed condition. acceptance. Initial deployment test was done with hydraulic energy replacing the pyro-thruster to simulate the thruster energy and to study the dynamic behaviour of the deployment system. Figure 13 shows CMF with composite grid fins in deployed condition.

\section{Flight performance}

Strain gauge instrumentation was provided on grid fin interfaces to assess the performance during flight. Grid fin deployment was monitored using high-speed cameras and a smooth deployment action was confirmed. Measured strain data during flight were comparable to that in grid fin acceptance tests. Composite grid fins were deployed successfully at CES activation and flight performance was as expected by the design.

\section{Conclusion}

Composite grid fin has been designed and developed for CES in Pad Abort Test. The design of grid fin is validated through structural acceptance test, correlating the measured strains and displacements with prediction. Realized grid fin has undergone hydraulic and pyro deployment tests meeting the functional requirements. The challenges in designing and realizing the grid fin with minimum weight and reasonable fabrication lead time are met through the application of advanced composite techno$\log$.

1. Simpson, G. M. and Sadler, A. J., Lattice controls: a comparison with conventional, planar fins, High Speed and Weapon Aerodynamics Department, Defence Evaluation and Research Agency, Bedford.

2. Jones, R. M., Mechanics of Composite Materials, Taylor \& Francis, USA, 1999, 2nd edn.

3. Kaw, A. K., Mechanics of Composite Materials, Taylor \& Francis Group, LLC, New York, 2006, 2nd edn.

4. Structural Materials Handbook, Vol-1, Polymer Composites, ESA PSS-03-203, Issue 1, Rev 1, 2003.

ACKNOWLEDGEMENTS. We thank the Director, VSSC, Thiruvananthapuram for permission to publish this paper. We also thank S. Sridhar (DD, STR; former Group Director, CLSG/CMSE) for support and encouragement during the course of design and development of the structure; all the agencies involved starting from the design phase till the induction of grid fin in Pad Abort Test flight, and colleagues of Composites Entity for their support.

doi: $10.18520 / \mathrm{cs} / \mathrm{v} 120 / \mathrm{i} 1 / 141-146$ 